\title{
Psychological Aspects in lifelike synthetic agents: Towards to the Personality Markup Language (A Brief Survey)
}

\author{
Maria Augusta Silveira Netto Nunes \\ Universidade Federal de Sergipe \\ Cidade Universitária 49100-000 - São Cristóvão-SE-Brazil \\ gutanunes@dcomp.ufs.br,
}

\begin{abstract}
This paper describes how human psychological aspects have been used in lifelike synthetic agents in order to provide believability during the human-computer interaction. We describe a brief survey of applications where Affective Computing Scientists have applied psychological aspects, like Emotion and Personality. Based on those aspects we describe the effort done by Affective Computing scientists in order to create a Markup Language to express and standardize Emotions. Because they have not yet concentrated their effort on Personality, here, we propose a starting point to create a Markup Language to express Personality.
\end{abstract}

Keywords: Lifelike Synthetic Agents, Emotions, Personality.

\section{Aspectos pedagógicos em agentes animados: Em direção a uma Markup Language para representar Personalidade}

Resumo. Esse artigo descreve como os aspectos humanos tem sido aplicados em Agentes Sintéticos Animados objetivando prover credibilidade durante a interação homem-máquina. Apresenta-se um breve estado da arte de pesquisas realizadas pelos cientistas em Computação Afetiva, pesquisas essas que incoporam aspectos psicológico, tais como Emoção e Personalidade. Considerando os aspectos discutidos, apresenta-se os esforços da comunidade científca em criar uma Markup Language para representar e padronizar Emoções. Considerando que os pesquisadores não tem ainda concentrado seus esforços na definição e padronização de Personalidade, esse artigo pretende ser um ponto de partida.

Palavras-chaves: Agentes Sintéticos Animados, Emoções, Personalidade.

\section{User Profile/Model In Affective Computing}

The aim of Affective Computing is to build computers that can recognize and respond to users Emotions and Personalities, simulate them and, finally, portray those ones. Affective Computing scientists are especially interested in developing Personality and Emotions in lifelike synthetic agents, as they are concerned about the importance of those psychological aspects used in agents so as to express their lifelike human character. They also believe that by enabling lifelike agents with Personality and Emotion through physiological and verbal actions during their social interaction might actually induce Emotion in others (humans using computer, for instance).

Affective Computing scientists have been trying to model human psychological aspects (mainly Emotions) since the 70's in order to implement what are believed to be lifelike agents, as seen in the works of Daniel Rousseau and Barbara Hayes-Roth (Rousseau and Hayes-Roth 1996), (Rousseau and Hayes-Roth 1998); Ortony et al (Ortony et al 1988), (Ortony et al 2002); Christine Lisetti (Lisetti 2002); Cristina Conati (Zhou and Conati 2003); Rosalind Picard (Picard 1997), (Picard 2002); James Lester (Lester et al 2000); Elliot (Elliot 1992); Ana Paiva (Paiva 2000), (Paiva and John 1995); Claude Frasson (Frasson and Gouarderes 1998), (Aivre et al 2002), (Ochs and Frasson 2004), (Chafar and Frasson 2004), amongst others.

Most of those works was intended to stimulate people's behavior by using a synthetic believable agent, which is considered "emotional". As people have psychologically answered to interactive computers as if they were humans (Reeves and Nass 1996), Affective Computing scientists have tried to model lifelike believable characters with Personality, goals and human-like emotions. The main aim to do it is because it contributes to coherence, consistency and predictability of computer emotional reaction and responses (Ortony 2002). The Personality of an agent can produce a performance that is motivated, believable and "theatrically" interesting for users (Rousseau 1996).

Affective Computing scientists use different technologies in order to try to extract those psychological aspects of the user and store them in User Profiles/Models. The main aspects included are: body responses; language and behavior changes (captured by approaches as recognition of emotions in speech and voice intonation); recognition of emotions in facial expressions; recognition of emotions in body gestures, posture or movement; physiological signals recognition (such as breathing, heartbeat rate, pulse, temperature, galvanic skin conductivity; eye tracing); and situation evaluation as described by 
Paiva (Paiva 2000). Unfortunately, those technologies are very intrusive and hard to be applied to conventional users in large scale (in web, for instance).

In the Affective Computing literature is proved that the main interest of scientists is being towards to define, model and extract the user Emotions in order to use those aspects to improve the interface and the interaction human-computer. That happens because people treat computer like humans. In fact, people seem to use similar social rules to interact with computers and other people. In particular, people perceive computers as having personalities (Reeves and Nass 1996).

Considering yet that Human Personality, together with Emotions, is a very important psychological characteristic considered during human reasoning and the decision-making (Damásio 1994), (Damásio 1999), (Simon 1983), (Goleman 1995), (Paiva 2000), (Picard 1997), (Picard 2002), (Trappl 2002), (Thagard 2006). Indeed, considering that Personality implies Emotions and many Affective Computing scientists have been incorporating, partially, Personality Traits in their modeling of lifelike emotional believable agents. And finally, considering that all effort from Affective Computing scientists has been done toward to standardization to Emotions ${ }^{1}$. We decide to fill the gap left by them by proposing the creation of a drive force towards to the standardization of the representation of Personality by using a Markup Language.

Thus, this paper describes the research done by Affective Computing scientists that have been using both Emotions and Personality in order to reach believability in their lifelike synthetic agents. Followed by the next section where the state of the art of Personality is presented. Finally we conclude with directions about how to formalize and represent Personality in virtual or real agents in order to be able to reach more believability for human-computer interaction.

\section{Affective Computing Applications}

To look believable, an agent needs to incorporate a deeper model of Personality and Emotions and, in particular, connect these two concepts together (Andre et al 2000). Psychological aspects characterize all variables that influence the behavior of an individual (virtual or not). According to Rousseau and HayesRoth (Rousseau and Hayes-Roth 1996), a set of psychological traits that make an individual unique and give him or her a style, is called Personality.

Considering this, some applications designed by Affective Computing scientists that include, mainly, aspects of Personality are presented next:

- [Rousseau and Hayes-Roth] (Rousseau and Hayes-Roth 1996) decided to create a synthetic character with Personality. This character considers an actor in a specific plot scenario. It evolves according to his actions and his predefined character's aspects. Characters are defined with few Personality Traits, which influence their behavior. The action that an actor may perform happens according to his Personality Traits and moods (temporary psychological states). By using Personality traits (confidence, activity and friendliness based on Cattel's work (Cattel 1945) in synthetic agents, Rousseau and Hayes-Roth intended to create an emotional impact on a human being who is interactive on the plot.

- $\quad$ OOrtony et al]"In order to build truly believable emotional agents, we need to endow them with Personalities that serve as engines of consistency and coherence rather than simply pulling small groups of traits out of the thin air of intuition" (Ortony 2002).

They have created a project of an inhabited market place where Personality Traits are used to modify characters' roles of virtual actors in sales situations. This project models Emotions and Personality Traits. Emotions based on OCC model (Ortony et al 1988) and Personality Traits based on Five Factor Model (McCrae and John 1992).

The Personality and Emotions are used as filters to constrain the decision process when selecting and starting the agent's behavior. In this project there are four agents. The user may activate no more than three at a time. The roles of the agent can be: seller, buyer 1 or buyer 2. Personality Traits (only 2 of 5 FFM are used - Agreeableness and Extroversion) are selected by the player. In the sales scenario, Emotions are essentially driven by the occurrence of events. The events are the speech act of the participant's dialogue that are evaluated by the characters in terms of their role, Personality Traits, and individual goals. The choice of dialogues acts is based on the actor's Personality while emotions are expressed by facial expressions.

\footnotetext{
${ }^{1}$ During the last years, many scientists have proposed non-standard markup languages to represent Emotions. According to W3C (W3C 2007), researchers in Emotion have neither validated those languages scientifically, nor they have been designed for general use in different application areas. Thus, finally, the W3C consortium decided to created a standard markup language of Emotions, called EmotionML (Emotion 2009) in order to standarlize Emotions.
} 
Personality Traits are used in order to personalize the agents' environment. Based on the agents' goals and Personality, the system may induce their dialogue and, therefore, their Emotion. Such information may change the Emotion and affective state of human, regarding computers.

- [Cristina Conati] (Zhou and Conati 2003) proposes the use of Personality Traits as a mechanism to help the inference of user's Emotions in an educational game. She believes that the user's Personality Traits should be connected to the user's goals to refine the affective User Model in the game.

She assesses students' Personality by using the 100-standard marker of Goldberg (Goldberg 1992). The test is based on the Big Five factors. The correlation between user's Personality Traits (extracted from the Goldberg test) and user's goals (extracted from user's action in the game) are deduced when users play the game. An example of the correlated interaction amongst users' Personality Traits and users' pattern goals finalized by the user's individual action in the game is presented in Figure 1.

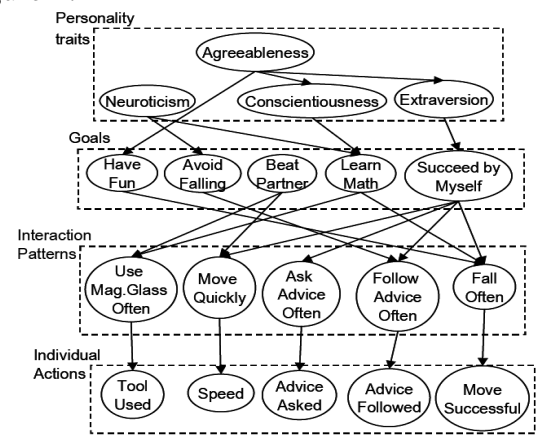

Figure 1. A model to assess goals (Conati 2003)

For the case described above, Conati presented the importance of psychological aspects in the decision-making process. She explained how the computer game responds intelligently to user's (student) Emotions. Firstly, the system interprets the user's actions, generating cues by mapping the user's Personality Traits into user's goals that lead to Emotions. She also stressed that very little work has been done towards an affective model considering Personality Traits. Many times Emotions are visualized and extracted based on physiological signals representing moods, which are short term or momentary Emotions. All in all, Personality Traits are sources of cues about how to produce Emotions (positive and negative) considering the most permanent aspects of the user.

- [Christine Lisetti] (Lisetti 2002) describes a scheme to represent psychological aspects to be used in the design of socially intelligent artificial agents. This scheme is composed of taxonomy of affection, mood, emotion, and Personality as presented in Figure 2.

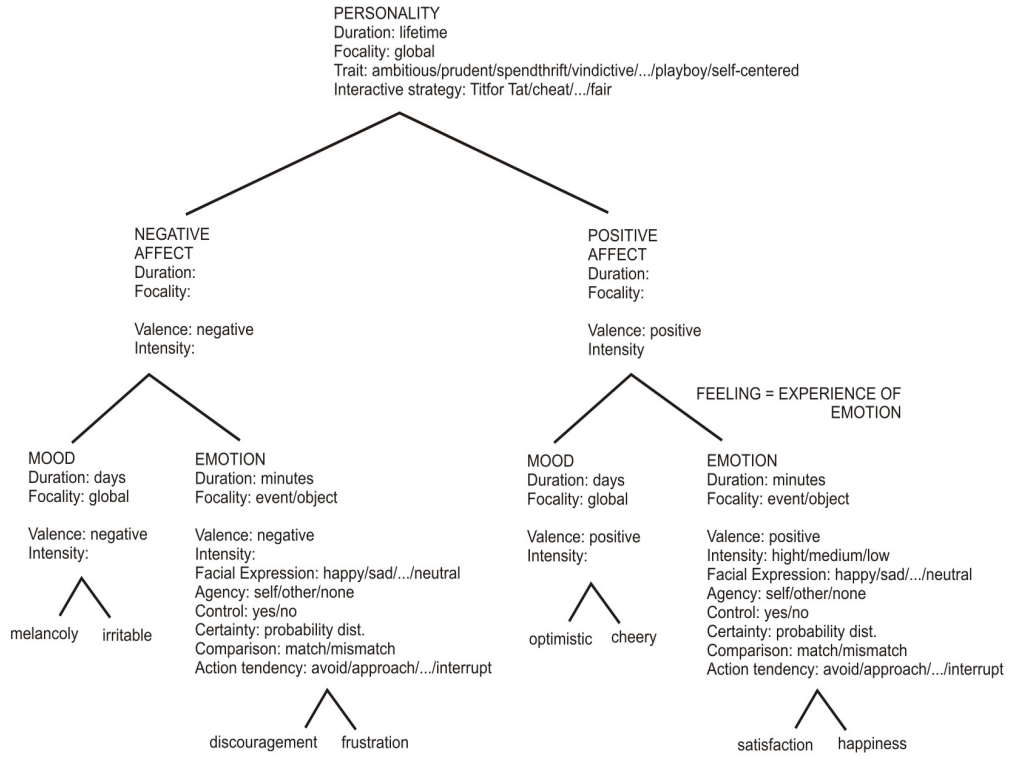

Figure 2. Hierarchical Model of Personality, Affect, Mood and Emotion (Lisetti 2002)

V. $7 \mathrm{~N}^{\mathrm{o}}$ 3, dezembro, 2009 
In Figure 2, Personality is at the top of the hierarchical model, which means that, in a system agents with different Personality types might experience the full range of possible Emotions. Those Emotions are related to goals and agents' actions tendencies in the system. As Emotions are at the bottom of the hierarchy, they do not necessarily imply Personalities. However, the contrary is true, that is, Personalities may influence Emotions. Personality has a different lifetime from Emotions. It is quite stable and it is not related to any specific event, unlike Emotions that are changeable appearing as consequence of an action and, consequently, generating a positive or a negative attitude. Different Personalities may produce different categories of Emotions. As Personality is at the top, the system can predict the user's needs and/or behavior.

This scheme proposed by Lisetti has demonstrated how crucial Personality is for the representation of human's affective aspects to be modeled by lifelike believable agents as well as to be used as cues to conduct research in other applications intended to use Personality.

- [Ana Paiva et al] at GAIPS (Intelligent Agents and Synthetic Characters Group)

- [Martinho and Paiva] (Martinho and Paiva 1999) present Tristão and Isolda, two dolphins that lived in the synthetic estuary of the river Sado during the EXPO98 ${ }^{2}$. They are virtual characters created to test and validate the viability of building the synthetic personae of believable emotional agents in intelligent virtual environments.

Researchers believe that virtual characters, like Tristão and Isolda are actually believable if they are consistent with the Personalities and Emotions they should represent. Personalities of Tristão and Isolda are identified by a set of emotional reactions in the river Sado. Personalities of characters are defined by using a Myers Bringgs (Myers 1962) model and the Big Five (John and Srivastava 1999). Tristão and Isolda have different Personalities that are identified by their emotional reaction in the environment. The Personality is defined in terms of temporal consistency and described in general terms, unlike Emotions, that correspond to a particular event defined in terms of temporal inconsistency.

Again, some psychological aspects such as Personality and Emotions are predefined in virtual agents. It helps them to be easily understood by humans, improving humancomputer interactions. This research corroborates that psychological aspects implemented in computers may benefit the user's comprehension and interaction with the modeled agents.

- FearNot! (Dias and Paiva 2005) is a system created to reduce bullying problems in schools. FearNot! presents John and Luke as colleagues. Luke does not like John and usually insults him. The aim of the system is to create emphatic characters to provide the illusion of life and the establishment of emotional relations between them. Luke and John are autonomous characters with distinct Personalities that direct their reasoning and behavior. That means, they may act differently in the same situations because of their Personalities. Personalities as well as Emotions are modeled according to the OCC (Ortony 1988) model. The Personality is specified by the character's goals, emotional reaction rules, action tendencies, emotional thresholds and decay rates. That model of Personality influences the character's action and also influences the generation of Emotions.

- Perfect Circle, developed by Rui Prada and Paiva (Prada and Paiva 2005), (Prada 07), (Prada and Paiva 2005a) (Prada and Paiva 2009) (Prada, Ma and Nunes 2009) is a game that supports the dynamics in a group of synthetic agents. The group dynamics considers socio-emotional and task-related interactions amongst synthetic agents. The game consists of four autonomous synthetic agents acting collaboratively in a believable group dynamics. The agents are engaged in the same goal. They should coordinate their actions to follow a similar strategy so as to reach their goal. The group members should, firstly discuss each action performed in the game for the resolution of the task.

Different members of the group have different skills to be applied so that the task is solved collaboratively. Each group member is characterized considering a set of attributes that differentiate the Personality of each one. The Personality of the agents is defined by two out of five dimensions of the Five Factor Model (Big Five), Extraversion and Agreeableness. The agents' Personality influences the interaction amongst them towards the task resolution.

${ }^{2}$ EXPO98 is a world exhibition held in Lisbon in 1998. V. $7 \mathrm{~N}^{\mathrm{o}}$ 3, dezembro, 2009 
This example is more related to a group dynamics than to psychological aspects, even if Personality is the key for the creation of believability in a group dynamics.

In order to better use and apply user's Personality in wherever other computer environment, we should firstly have a better understanding about what Affective Computing scientists have done towards modeling virtual embodied emotional agents. Based on this study, we have extracted cues on how they have modeled agents' psychological aspects (real/virtual), even if we have been particularly interested in Personality.

The Affective Computing scientists are neither proposing newer Personality nor Emotional theories. They have only used the available ones in order to drive agents' goals. By recovering human Personality and modeling them in computers, we enable computers to manipulate their own decisionmaking process. As we described before, decision-making is essential during the personalization of a virtual environment.

Considering this, next we present the state of the art of Personality extracted from Psychology. We also present the perspective about how we could model, formalize and assess human Personality to be use in computers in order to improve the human-computer interaction. We believe that our study could be a starting point towards to the creation of a Markup Language to express Personality.

\section{Towards Personality Markup Language}

\subsection{Representing Personality as a Psychological aspect}

Personality does not have a common definition. According to Schultz (1990) in its Latin origin, the word Personality "Persona" refers to a mask used by an actor in a play to show his appearance to the public.

Schultz extends his definition describing Personality as "an enduring and unique set of characteristics that does not have any chance in response to different situations". Indeed, Burger (2000), defines it as "consistent behavior patterns and intrapersonal processes originating within the individual".

We know that Personality is more than just superficial physical appearance. Personality is relatively stable and predictable. However, Personality is not rigid and unchanging, it is normally kept stable over a 45-year period which begins in young adulthood (Soldz 1998). According to psychologists Personality definitions can be better defined based on the theory/approach of Personality that it belongs to.

Theories of Personality were created to ease individual understanding of oneself and others (Cartwright 1979). There are more than 18 theories of Personality described by researchers. Each one describes alternative ways to present and differentiate human Personality. According to Schultz (1990) they can be grouped in 9 categories: Psychoanalytic, Neopsychoanalytic, Trait, Life-spam, Humanistic, Cognitive, Behavioral, Social-learning and Limited-domain. Alternatively, Funder (2001) and Burger (2000) also propose other categorization approaches: Trait approach, Biological approach, Psychoanalytic approach, Phenomenological/Humanistic approach, Behavioral approach and Cognitive approach.

Each theory/approach of Personality focuses on how Personality is used and defined by psychologists and how each approach differ from one another in terms of conceptions and measures. Psychologists (Burger 2000) (Funder 2001) who agree with the Psychoanalytic approach argue that people's unconscious minds are largely responsible for important differences in their styles of behavior. In the Trait approach, psychologists focus their efforts on the ways people differ psychologically from one another and how these differences might be conceptualized or measured (Personality Traits). Psychologists using the Biological approach, point to inherited predispositions and physiological processes to explain individual differences in Personality. In the Phenomenological/Humanistic approach, personal responsibility and feelings of self-acceptance are identified as key causes of differences in Personality. Psychologists who adhere to the cognitive approach conduct experiments on how the basic cognitive processes of perception, memory, and thought affect behavior and Personality. The Behaviorist/Learning approach focuses on behavior and ways in which it can be affected by rewards and punishments.

We propose to use the Traits approach because it is the approach with which we can psychologically differentiate people by using conceptualization and measurable traits, called Personality Traits. Indeed, Personality Traits is a set of human features that can be easily modeled and implemented in computers.

\subsection{The Trait approach}

The Trait approach describes the psychological differences amongst individuals. It is based on empirical research. Personality Traits were first studied and defined by Gordon W. Allport (Allport and Allport 1921), (Allport 1927). Allport studied Personality based on healthy people as opposed to his colleagues who studied abnormal and pathological Personalities (Schultz 1990). He created 17.953 traits to describe the Personality of an individual (Funder 1991). Allport believes that every human is unique. He describes 
each human as having common and individual traits. Therefore the intensity of those traits will be forcedly different (Barkhuus and Csank 1999). That means, for instance, that Mary and Jane may be both "aggressive" people, although the range of aggressiveness of each one will be different. That difference comes from their individual history and never-repeated external/environmental received influences. Thus, even if Mary and Jane have the same trait (aggressiveness) the intensity will not be the same.

Allport defines common traits as those shared amongst many people within a culture, measurable on a scale. On the other hand, individual traits are traits that refer just to personal dispositions, unique in an individual ${ }^{3}$. 17.953 traits defined by Allport include common traits as well as individual traits. Kuckhohn and Murray (1953) agree with Allport when he says that "every man is: like all other men, like some other men and like no other men". As most individual differences are meaningless in people's daily interactions, in order to limit the definitions of traits in an exponential way, otherwise growing exponentially thus becoming intractable, researchers assume that the trait approach is based on the idea that all men are "like some other men" (Funder 1991).

In this regard, Cattel proposes a subset of Allport traits. He proposes 4.500 traits items against the 17.953 created by Allport. Those 4.500 were correlated to 171 scales after some empirical analysis (Goldberg 1990). After, Cattel reduced an extra 99\% of those items transforming them into 35 bipolar sets of related items, which were factor analyzed ${ }^{4}$. As a consequence, he identified 12 Personality factors. They were analyzed by orthogonal rotational methods, which proved that only five factors were replicable (Goldberg 1990), as a result, the "Big Five" Model was created.

The formal beginning of the Big Five (John and Srivastava 1999)/FFM ${ }^{5}$ (Five Factor Model) (Mcrae and John 1992) was created by Fiske, replicated by Norman and derived from Cattel's natural language traits. They were initially numbered and labeled as: (i) Surgery (or Extraversion), (ii) Agreeableness, (iii) Conscientiousness (or Dependability), (iv) Emotional Stability (vs. Neuroticism), (v) Culture. Afterwards, researchers (Mcrae and John 1992) readapted the labels ${ }^{6}$ to: (i) Extraversion, (ii) Agreeableness, (iii) Conscientiousness, (iv) Neuroticism, (v) Openness to Experience.

Essentially, to simplify and organize the traits, researchers created the Big Five model. On the other hand, researchers asked one another if only five traits were sufficiently accurate to measure Personality differences. According to John and Srivastava (1999):

"The Big Five structure does not imply that Personality differences can be reduced to only five traits. Yet, these five dimensions represent Personality at the broadest level of abstraction, and each dimension summarizes a large number of distinct, more specific Personality characteristics".

In order to make it clearer, we cite an example given by Norman. He created a pool of 2.800 trait items, which were applied to university students. From those items, after the test application, he classified 1.431 items grouped into 75 categories called factor-pole, then he re-grained into 5 "Big Five" factors (Goldberg 1990). Even if Big FivelFFM factors represent a broad level of Personality structure, they do not guarantee the exhaustion of all significant Personality dimensions.

Aiming to distinguish factors and facets and their levels of specificity versus generality, De Raad and Perugini (2002) describe two approaches called hierarchical and circumplex: (i) The first one defines facets as first order factors and the Big Five as second order factors. For instance, NEO-PI-R (Costa and McCrae 1992) has 6 facets for each factor (5)); (ii) The second one is a finer-grained configuration distinguishing 90 segments in the AB5C (Abridged Big five Circumplex) (Hosfstee et al 1992). Normally each facet consists of two different factors.

Our study focuses on the first approach because the definition of facets is simpler and it is much more used than the others.

Facets are used by psychologists in order to enrich Big Five dimensions with more fine-grained characteristics. To illustrate this, we present, in the Table 1, the 5 Big Five dimensions followed by their correspondent facets.

\footnotetext{
3 that means, just a few people have this trait.

4 "The factor analytic technique is designed to identify a group of things - such as test items - that seem to be alike" (Funder 1991).

5 "The term Big Five was coined by Lew Goldberg and was originally associated with studies of Personality Traits used in natural language derived from lexical data (Saucier and Goldeberg 1996 and based on empirical phenomenon. The term Five-Factor Model, which has been more commonly associated with studies of traits using Personality questionnaires" (Srivastava 2006).

6 Other names are also given to each of the factors. Neuroticism is often referred to as Negative Affectivity; Extraversion is also known as Social Activity; Agreeableness is sometimes referred to as Affection or Socialization; and Conscientiousness is also known as Will to Achieve" (Nass et al 1995).
}

V. $7 \mathrm{~N}^{\mathrm{o}}$ 3, dezembro, 2009 
Table 1. NEO-PI_R Facets of Big Five (extracted from (Jonh and Srivastava 1999)

\begin{tabular}{|c|c|}
\hline ग9ว6' & 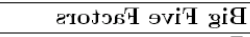 \\
\hline 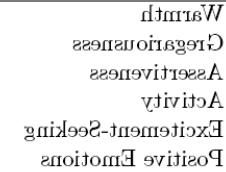 & srosergesnotsit \\
\hline 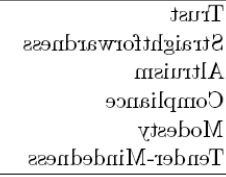 & zеssrgلd \\
\hline 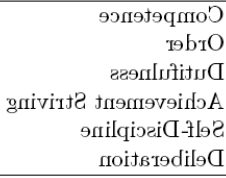 & 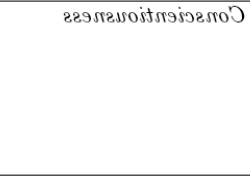 \\
\hline 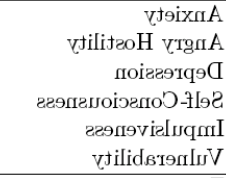 & stresistorssg $\mathrm{V}$ \\
\hline 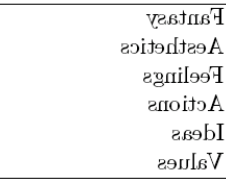 & 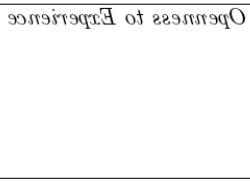 \\
\hline
\end{tabular}

In order to extract human traits (as Big Five factors and their respective facets) psychologists usually use computer-based questionnaires. Those questionnaires are directly applied by psychologists, or may be freely available on the web. They might have either a large or a small amount of questions. The number of questions in the questionnaire is directly related to the granularity of the desired extracted traits from each person's Personality. Questionnaires are called Personality Tests and are described next.

\subsection{Personality Test}

A Personality Test is a computer narrative ${ }^{7}$ that generally reveals an established set of traits of the individual that differentiates one from another human being. Johnson's (1994) defines it as

"a report based on empirical research that can tell a test-taker how someone's Personality is likely to influence job performance, health, relationships and other significant life events, being useful to provide insights and to make predictions about individuals".

Researchers propose a wide range of instruments to assess human Personality Traits. For instance 16PF (Cattell's 16 Personality Factors Questionnaire) and 6FPQ (Six Factor Personality) are based on other constructions, different from the Big Five. Therefore, we are particularly interested in Personality Tests based on 5 constructions (Big Five) because it is more largely used by scientists, psychologists and business test-appliers and test-takers (Costa and McCrae 1992), (Jonhson 1994), (Saucier and Goldberg1998).

After analyzing a list of Inventories (Nunes 2009) we hypothesized that the number of items influence the precision of the traits measured. The bigger the number of items, the finer grouped and more accurate the extracted traits will be.

Nunes (Nunes 2009) describes that NEO-PI-R is different from most other inventories. That is because it assesses 5 factors of BIG FIVE including also 6 more facets for each dimension (30 facets in total) using then a fine-grained description of people's Personality Traits and, consequently, a bigger precision in those representations of traits.

NEO-PI-R (Costa and McCrae 1992) is also defined as one of the most robust, used and wellvalidated commercial inventory in the world (Johnson 2000b), (Johnson 2005). It has been used in over a thousand published studies where it demonstrated longitudinal stability, predictive utility, and consensual validation (Costa et al 2002). The NEO-PI-R is a commercial inventory and, consequently, a proprietary instrument, (as most of broad-bandwidth Personality inventories) its items are copyrighted and cannot be used freely by other scientists.

Alternatively, Goldberg has proposed in collaboration with researchers from the Rijksuniversiteit Groningen (The Netherlands) and Universitat Bielefeld (Germany), the creation of a public domain scale

7 also called inventory, questionnaire or adjective scale.

$$
\text { V. } 7 \mathrm{~N}^{\mathrm{o}} \text { 3, dezembro, } 2009
$$


called IPIP - The International Personality Item Pool (Goldberg 1999). The IPIP, according to the IPIP consortium website (IPIP 2009), is defined as "a Scientific Collaboratory for the Development of Advanced Measures of Personality and Other Individual Differences".

According to Johnson (Johnson 2001) (Johnson 2000) the IPIP Consortium created a set of 1252 items in IPIP. Goldberg's research team has been able to identify, empirically, sets of IPIP items that measure the same constructions as commercial inventories. Scales formed from these items sets possess psychometric properties that match or exceed those of the original commercial scales. In order to find a taxonomic framework to organize the nearly countless variety of individual differences that might be measured, IPIP also uses a BIG FIVE factor structure as NEO-PI-R does.

NEO-IPIP Inventory (Johnson 2000) (Johnson 2005) appeared when Johnson chose from the various Personality inventories at Goldberg's IPIP Website (IPIP 2009) with his 300 items proxy for the revised NEO Personality Inventory (NEO-PI-R) (Costa and McCrae 1992). Johnson decided to create an IPIP-NEO because it is a free-of-charge version of NEO-PI-R which is, as previously described, one of the most robust, known and well-validated commercial inventories in the world (Johnson 2000) and also because it is based on Five-factor or Big Five dimensions. NEO-IPIP Inventory was used and wellvalidated by Johnson (Johnson 2000b), (Johnson 2005). From August 1999 to May 2001, 175000 people answered the online NEO-IPIP questionnaire. Then, 21588 answered questionnaires were selected as a valid protocol (Johnson 2001)

In Nunes (2009) we have been using the NEO-IPIP Inventory in order to conceive, model, formalize and implement a Psychological User Profile/Model. That model of the Psychological User Profile was applied in experiments conducted by Nunes, described in (Nunes 2009) and (Prada, Ma and Nunes 2009). Those experiments gave us promising results which incentive us to propose further studies in the representation of Personality towards to the creation of a Personality Markup Language.

\section{Conclusions and Future Works}

As we see in the paper, the Affective Computing Scientists have been implementing Emotions explicitly. That happened because Emotions are more easily measurable and interpretable and they may influence directly user's action-interaction in computer environments. In fact, Emotions are instantaneous, they have a short life-time and change constantly, differently from Personality that is much more stable and, normally, kept stable in adulthood. Emotions and Personality when implemented and identified by computers may enable a better interaction and interface human-machine.

Considering the previous aspects discussed before, it is possible to say the following: Personality implies emotions; every agent (real/virtual) who feels Emotions has a Personality; and, usually, Personality does not appear explicitly even if it influences Emotions directly. Because many applications in computers have already contemplated the formalization, the model and the implementation of Emotion (including the standardization proposed by W3C (Emotion ML 2009), we decided, this time, to give priority to Personality. Thus, in this paper we presented a brief survey about the theories of Personality, including different approaches and inventories, we also addressed how we could represent it real/virtual agents.

By recovering human Personality we allow computers to manipulate their own decision-making process enabling them to provide users with more diversified and personalized computer environments. Personality enables computer to decide what type of resource it could apply in order to better personalize the environment especially to people needs. Different from Emotions that enables computers to better know people's emotional state (mood, for instance) and so it could decide if they will present or not the personalized environment selected before considering people' personality.

Our future works includes to found partners in order to start the studies for the creation of a W3C Markup Language for representing Personality. We have conducted many researches towards to model Personality, they are: we have been using the Psychological aspects in areas like Distance Education (Nunes et al 2009c), Twitter (Nunes and Aranha 2009b), Virtual Games (Prada, Ma and Nunes 2009).

\section{References}

Allport, F. and Allport, G. Personality traits: Their classification and measurement. Journal of Abnormal and Social Psychology, (16):6-40, 1921. (Available at http://psychclassics.yorku.ca/Allport/Traits/).

Allport, G. Concepts of trait and personality. Psychological Bulletin, (24):284-293, 1927.

Andre, E. et al. Integrating models of personality and emotions into lifelike characters. pages 150-165, 2000.

Barkhuus, L. and Csank, ,P. Allport's theory of traits - a critical review of the theory and two studies. Technical report, Concordia University, 1999. (Available in http://www.it-c.dk/ barkhuus/allport.pdf).

Buchanan, T. et al. Implementing a five-factor personality inventory for use on the internet. European Journal ofPsychological Assessment, 21(2):116-128, 2005.Cattell, R. The description of personality: Principles and findings in a factor analysis. The American Journal of Psychology, 58(1):69-90, 1945.

$$
\text { V. } 7 \mathrm{~N}^{\mathrm{o}} \text { 3, dezembro, } 2009
$$


Cartwright, D. Theories and Models of Personality. WCB, 1979.

Chaffar, S. and Frasson, C.. Inducing Optimal Emotional State for Learning in Intelligent Tutoring Systems. In J.C. Lester et al., editor, Intelligent Tutoring Systems-ITS04, 3220, pages 45-54, Berlin, 2004. Springer-Verlag.

Costa, P.; McCrae, R. and Jonsson, F.. Validity and utility of the Revised NEO Personality Inventory: Examples from Europe. In B. De Raad and M. Perugini, editors, Big Five Assessment, chapter 3, pages 61-77. Hogrefe Huber, Germany, first edition, 2002.

Damasio, A.R. Descartes' Error: Emotion, Reason, and the Human Brain. Quill, New York, 1994.

Damasio, A. R. The Feeling of What Happens. Harcourt, Orlando, Florida, 1999.

De Raad ,B. and Perugini, M.. Big Five Factor assessment: Introduction. In B. De Raad and M. Perugini, editors, Big Five Assessment, chapter 1, pages 1-26. Hogrefe Huber, Germany, first edition, 2002.

Dias, J. and Paiva, A.. Feeling and reasoning: A computational model for emotional characters. In EPIA, pages $127-$ 140, 2005.

Emotion Markup Language (EmotionML) 1.0 -W3C, 2009 (available at http://www.w3.org/TR/emotionml/ )

Elliot,C. The Affective Reasoner: A Process Model of Emotions in a Multi- Agent system. Phd thesis - technical report 32, Institute for the Learning Sciences - Northwestern Univeristy, Evanston-Illinois, June 1992. (Available at http://condor.depaul.edu/ elliott/ar/ftp/elliott-thesis.pdf.

Faivre, J., Nkambou, R. and Frasson. C. Integrating Adaptive Emotional Agents in ITS. In S.A. Cerri, G. Gouardéres, and F. Paraguaçu, editors, Intelligent Tutoring Systems-ITS02, 2363, pages 996-997, Berlin, 2002. Springer-Verlag.

Funder, D.. The Personality Puzzle. Norton, second edition, 2001.

Frasson, C. and Gouarderes, G.. Workshop II - Pedagogical Agents. In B.P. Goettl et al., editor, Intelligent Tutoring Systems-ITS 98, 1452, page 662, Berlin, 1998. Springer-Verlag.

Goleman, D. Emotional Intelligence - Why it can matter more than IQ? Bloomsbury, London, first edition, 1995.

Goldberg, L.. An alternative "Description of Personality": The Big-Five Factor structure. Journal of Personality and Social Psychology, 59(6):1216-1229, 1990.

Goldberg, L. A broad-bandwidth, public-domain, personality inventory measuringthe lower-level facets of several five-factor models. Personality Psychology in Europe, 7:7-28, 1999.

Goldberg, L.. The development of markers for the big-five factor structure. Psychological Assessment, 4:26-42, 1992.

Hofstee, W. K. B.; De Raad, B. and Goldberg. L. R. Integration of the Big Five and circumplex approaches to Trait structure. Journal of Personality and Social Psychology, 63(1):146-163, 1992.

IPIP The international Personality item pool, 2009. (Available at http://ipip.ori.org/ipip/).

Johnson, John A... Computer narrative interpretations of individual profiles. R. Hogan, J. Johnson and S. Briggs, 1994. (. Available from the author at Penn State DuBois, DuBois, PA 15801.).

Johnson, John A.. Web-based personality assesment. In 71st Annual Meeting of the Eastern Psychological Association, Baltimore, USA, 2000. (Available at http://www.personal.psu.edu/ j5j/vita.html).

Johnson, John A.. Predicting observers ratings of the big five from the cpi, hpi, and neo-pi-r: A comparative validity study. European Journal of Personality,14:1-19, 2000 b.

Johnson, John A.. Screening massively large data sets for nonresponsiveness in web-based personality inventories., 2001. Invited talk to the joint Bielefeld-Groningen Personality Research Group, University of Groningen, The Netherlands.(Avialable at http://www.personal.psu.edu/faculty/j/5/j5j/papers/screening.html).

Johnson, John A.. Ascertaining the validity of individual protocols from webbased personality inventories. Journal of research in Personality, 39(1):103-129, 2005.

John, O. and Srivastava, S.. The big five trait taxonomy: History, measurement, and theoretical perspectives. In Lawrence A. Pervin and Oliver P. John, editors, Handbook of Personality: Theory and Research, pages 102-138. The Guilford Press, New York, second edition, 1999.

Kluckhohn, C. and Murray, H. Personality Formation: The determinants. In Clyde Kluckhohn and Henry A. Murray with the collaboration of David M. Schneider, editors, Personality in Nature, Society and Culture, chapter Two, page 53. Alfred A. Knopf, New York, second edition, 1953.

Lester, J. et al. Deictic and emotive communication in animated pedagogical agents. pages 123-154, 2000.

Lisetti, C.. Personality, affect and emotion taxonomy for socially intelligent agents. In Proceedings of the Fifteenth International Florida ArtificialIntelligence Research Society Conference, pages 397-401. AAAI Press, 2002.

Martinho, C. and Paiva., A. "underwater love": Building trist ao and isolda 'spersonalities. In Artificial Intelligence Today, pages 269-296. 1999.

Mcrae, R and John,O.. An introduction to the five-factor model and its applications. Journal of Personality, 60(2):175-216, June 1992.

Myers, I. The Myers-Briggs Type Indicator. Palo Alto, CA:Consulting Psychologists Press, 1962.

Nass, C. et al. Can computer personalities be human personalities? International Journal Humuan-Computer Studies, 43(2):223-239, August 1995.

V. $7 \mathrm{~N}^{\circ}$ 3, dezembro, 2009 
Nunes, M. A. S. N. . Recommender Systems based on Personality Traits:Could human psychological aspects influence the computer decision-making process?. 1. ed. Berlin: VDM Verlag Dr. Muller, 2009. v. 1. 140 p.

Nunes,M.A.S.N.; Aranha, C. Tendências à Tomada de Decisão computacional. In $1^{\circ}$ Conferência W3C Brasil. São Paulo.2009b; (to appear)

Nunes, M.A.S.N et al. Repensando os ambientes virtuais de aprendizagem: o caso da UFS. In XX SBIE: II Workshop sobre Modelos Pedagógicos em Educação a Distância. SBC 2009c. (submitted paper)

Nunes, M. A. S. N. ; Cerri, Stefano A. ; Blanc, N. . Improving recommendations by using Personality Traits in. In: International Conference on Knowledge Management-, 2008, Graz-Austria. I.KNOW08, 2008. v. 1. p. 92-100.

Ochs, $\mathrm{M}$ and Frasson, C. Optimal Emotional Conditions for Learning with an Intelligent Tutoring System. In J.C. Lester et al, editor, Intelligent Tutoring Systems-ITS04, 3220, pages 845-847, Berlin, 2004. Springer-Verlag.

Ortony, A. On Making Believable Agents Believable. In R. Trappl, P. Petta, and S. Payr, editors, Emotions in humans and artefacts, chapter 6, pages 189-211. A Bradford Book - MIT Press, Cambridge, Massachusetts, 2002.

Ortony, A and Clore, G.and Collins, A.. The Cognitive Structure of Emotions. Cambridge University Press, USA, 1988.

Paiva, A. and Self, J.. Tagus - a user and leamer modeling workbench. User Modeling and User-Adaptation Interactions, 4(3):197-226, 1995.

Paiva, A. Affective interactions: towards a new generation of computer interfaces. pages 1-8, 2000.

Picard, R.. Affective computing. MIT Press, Cambridge, MA, USA,1997.

Picard, R. What does it mean for a computer to 'have' Emotions? In R. Trappl, P. Petta, and S. Payr, editors, Emotions in humans and artefacts,chapter 7, pages 213-235. A Bradford Book - MIT Press, Cambridge, Massachusetts, 2002.

Prada, R and Paiva, A. "Believable groups of synthetic characters," in AAMAS '05: Proceedings of the fourth international joint conference on Autonomous agents and multiagent systems. New York, NY, USA: ACM, 2005, pp. $37-43$.

Prada, R., MA, S., Nunes, M. A. S. N. Personality in Social Group Dynamics In: International Conference on Computational Science and Engineering- CSE '09, 2009, Vancouver. International Conference on Computational Science and Engineering- CSE '09. , 2009. v.4. p.607 - 612.

Prada,R. and Paiva, A. "Teaming up humans with autonomous synthetic characters,"Artificial Intelligence, vol. 173, no. 1, pp. 80-103, 2009.

Prada, R; Otero, N. and Paiva, A. The user in the group: evaluating the effects of autonomous group dynamics. In ACE '07: Proceedings of the international conference on Advances in computer entertainment technology, pages 2532, New York, NY, USA, 2007. ACM.

Prada, R. and Paiva, A.. Synthetic group dynamics in entertainment scenarios:creating believable interactions in groups of synthetic characters. In ACE '05:Proceedings of the 2005 ACM SIGCHI International Conference on Advances incomputer entertainment technology, New York, NY, USA, 2005. ACM.

Reeves, B. and Nass, C. The media equation: how people treat computers, television, and new media like real people and places. Cambridge University Press, New York, NY, USA, 1996.

Rousseau, D. and Hayes-Roth, B. Personality in synthetic agents. Technical Report KSL-96-21, July 1996.

Rousseau, D. and Hayes-Roth, B. A social-psychological model for synthetic actors. In AGENTS '98: Proceedings of the second international conference on Autonomous agents, pages 165-172, New York, NY, USA, 1998. ACM Press.

Saucier, G. and Goldberg, L.. The language of Personality: Lexical perspectives on the five-factor model. In J. S. Wiggins, editor, The five-factor model of Personality: Theoretical perspectives, chapter 2, pages 21-50. Guilford, New York, 1996.

Saucier ,G. and Goldberg, L. R.What is beyond the Big Five? Journal ofPersonality, 66(4):495-524, August 1998.

Schultz, D. Theories of Personality. Brooks/Cole, forth edition, 1990. Jerry M. Burger. Personality. Wadsworth, fifth edition, 2000.

Simon, H.A. Reason in Human Affairs. Stanford University Press, California, 1983.

Soldz, S. and Vaillant, G.. The big five personality traits and the life course: A 45 years longitudinal study. Journal of Research in Personality, 33:208-232,1998.

Srivastava, S. Measuring the big five personality factors., 2006.

Thagard, Paul. Hot Thought: Machanisms and Applications of Emotional Cognition.A Bradford Book- MIT Press, Cambridge, MA, USA, 2006.

Trappl, Robert; Payr, Sabine and Petta, Paolo editors. Emotions in Humans and Artifacts. MIT Press, Cambridge, MA, USA, 2003.

W3C - emotion incubator group, 2007. (Available at http://www.w3.org/2005/Incubator/emotion).

Zhou, X. and Conati, C. Inferring user goals from personality and behavior in a causal model of user affect. In IUI '03: Proceedings of the $8^{\text {th }}$ international conference on Intelligent user interfaces, pages 211-218, New York, NY, USA, 2003. ACM Press. 
V. $7 \mathrm{~N}^{\mathrm{o}}$ 3, dezembro, 2009 